Lunge sonst normal. Probepunktion im linken achten Interkostalraum hinten zeigt klare, seröse Flüssigkeit.

Ich ordinierte Bettruhe unter Behandlung des Hausarztes. In den folgenden Jahren besuchte die Patientin mich dreimal in meiner Sprechstunde; sie war nicht zu Bett gegangen, hatte im Gegenteil die ganze Zeit von August 1903 bis April 1907 ihr Geschäft besorgt, ohne einen Tag zu versäumen. Bei den Untersuchungen konnte ich nur Residuen einer alten, schweren Pleuritis finden. Im Frühling 1907 trat indessen wieder Husten auf, außerdem Nachtschweiß und Abmagerung. Am 6. April 1907 konnte ich zahlreiche Rasselgeräusche über dem linken Apex bis zur zweiten Rippe vorne und bis zur Mitte der Regio infraspinata hinten konstatieren. Die rechte Lunge gesund. Im Sputum keine Tuberkelbazillen nachweisbar. - Kurz nachher wurde die Expektoration sehr reichlich, aber nur zeitweise; jeden Vormittag expektorierte die Kranke im Laufe von $1 / 2-1$ Stunde ein $100-120 \mathrm{ccm}$ dünnes, gelbes, nicht stinkendes Sputum; beim Vornüberbücken kam das Expektorat "ganz von selbst". Diese reichlichen Expektorationen wurden nach und nach seltener, soda $\beta$ die Patientin, als ich sie am 2. August 1907 wiedersah, in den letzten 6 Wochen nur zweimal diese Massenexpektorationen durchgemacht hatte. Auch jetzt war sie nie bettlägerig gewesen.

Status am 2. August 1907: Afebril. Puls 80, R. 22. Linke Thoraxhälfte etwas abgeflacht, beinahe unbeweglich während der Respiration. An der linken Vorderfläche starke Dämpfung von der Spitze bis zur dritten Rippe mit tympanitischem Beiklang über der Clavicula und im ersten Interkostalraum; nach unten geht diese Dämpfung in die Herzdämpfung über. In der Axillarfläche voller sonorer Schall. In der Fossa supra- und infraclavicularis ist die Inspiration stark abgeschwächt, die Exspiration hauchend; außerdem feine Sibili und recht zahlreiche feine und gröbere Rasselgeräusche, von welchen einzelne metallisch klingen. In der Axillarfläche ist die Respiration nur wenig abgeschwächt. Links hinten Dämpfung in der Fossa supraspinata, dagegen nicht in der infraspinata; am Angulus fängt wieder eine leichte Dämpfung an, die an der Basis beinahe vollständig ist. In der Fossa supraspinata derselbe auskultatorische Befund wie in der Fossa supra- und infraclavicularis, doch keine metallisch klingenden Rasselgeräusche; in der Infraspinata ist die Inspiration ein wenig abgeschwächt, die Exspiration im oberen Teil verlängert, nach unten dagegen ungefähr normal. Vom Angulus ab ist die Inspiration schwächer, die Exspiration mehr und mehr bronchial; beide können aber bis zur Basis verfolgt werden.

Die rechte Lunge zeigt keine physikalischen Abnormitäten. Spitzensto $B$ undeutlich, Herzdämpfung beginnt an der dritten Rippe und dem linken Sternalrand, die Herztöne rein. Urin klar, hell; kein Eiweiß

Die Symptome von April 1907 und später - Hustenanfälle mit reichlicher Expektoration im Laufe von $1 / 2-1$ Stunde - deuteten mit Bestimmtheit darauf, daß irgendwo ein Hohlraum existierte, der sich von Zeit zu Zeit durch Husten entleerte; wegen des tympanitischen Schalls und einzelner metallisch klingender Rasselgeräusche in der linken Spitze vermutete ich hier eine Kaverne. Da die Krankheit bis dahin sehr chronisch verlaufen und die rechte Lunge immer noch gesund war, dachte ich, daß ich durch eine Operation diese

\title{
Pyopneumothorax tuberculosus ambulans von vierjähriger Dauer.
}

\section{Resektion der zweiten bis elften Rippe. Heilung.}

\author{
Von P. Bull, Chirurg in Christiania.
}

Fräulein Julie J., Modistin, geboren 1875, konsultierte mich zum ersten Male im August 1903. Zehn Geschwister, eines an Phthise gestorben, die anderen und die beiden Eltern leben und sind gesund. Sie selbst ist immer gesund gewesen, bis sie im Juli 1902 einen linkseitigen Lungenspitzenkatarrh bekam, weshalb sie sich in einem Tuberkulosesanatorium bis Februar 1903 aufhielt; im Sputum wurden einzelne Tuberkelbazillen nachgewiesen. Sie verließ die Heilanstalt ohne Besserung mit physikalisch nach weisbaren Veränderungen bis zur dritten Rippelinkerseits; der behandelnde Arzt stellte eine schlechte Prognose. Im März 1903 wurde sie wegen Fieber wieder bettlägerig; Anfang April, als sie noch zu Bett lag, bekam sie eines Tages plötzlich nach einer stundenlangen Hustenattacke gewaltige linkseitige Brustschmerzen. Der hinzugerufene Arzt fand ihren Zustand äußerst drohend; die Schmerzen wurden indessen nach und nach besser, und einen Monat später war die Patientin fieberfrei und bald außer Bett. Seit Mai ist sie immer kräftiger geworden und hat teilweise ihr Geschäft besorgt.

Status am 24. August 1903: Klein, blaß, lebhaft. Die linke Thoraxhälfte bewegt sich beinahe garnicht während der Respiration. Absolute Dämpfung über der ganzen linken Brusthälfte mit Ausnahme der Fossa supra- und infraclavicularis, wo der Schall gedämpft tympanitisch ist. Stark abgeschwächtes Atmen über dem Apex, fernes Bronchialatmen unterhalb des Angulus, speziell während des Exspiriums. In der rechten Fossa supra- und infraspinata einzelne seltene Sibili, die rechte Kaverne zur Heilung bringen könnte. Um die Diagnose und die Lokalisation $\mathrm{zu}$ bestätigen und genauer $\mathrm{zu}$ fixieren, ließ ich am nächsten Tage meine Patientin mit Röntgenstrahlen durchleuchten, fand aber zu meiner großen Ueberraschung keine Lungenkaverne, sondern in der linken Pleurahöhle leicht bewegliche Flüssigkeit, deren Oberfläche in der Höhe der siebenten bis achten Rippe lag; in der linken Spitze unregelmäßige Verdichtungen des Lungengewebes bis zur dritten Rippe.

Ich mußte jetzt die Diagnose auf Pne u mothorax stellen, und zwar - nach der ganzen Entwicklung der Krankheit auf Pneumothorax tuberkulösen Ursprungs. Durch eine abermalige, exaktere Untersuchung konnte ich nun feststellen, daß die Patientin im April 1903 eine Woche nach dem Auftreten der intensiven linkseitigen Brustschmerzen bemerkt hatte, daß durch jede Bewegung im Bette ein Plätschergeräusch, ,als ob sie in der Brust ein Gefäß voll von Wasser hätte", entstand. Dieses Geräusch war so laut, daß es auch von den Angehörigen gehört wurde. Nach und nach wurde es mehr undeutlich, und die Kranke kann sich jetzt nicht erinnern, ob es noch nach ihrem Aufstehen im Mai hörbar war. Es kann nach diesen bestimmten Angaben kaum ein Zweifel darüber sein, daß sie im April 1903 einen Pneumothorax mit nachfolgender seröser Pleuritis akquirierte. In dem Maße, als das Exsudat später zunahm, ging der Pneumothorax zurück, bis die Luft vollständig oder beinahe vollständig von der Flüssigkeit ersetzt war; dies ist wahrscheinlich der Fall gewesen, als sie mich zum 
erstenmal konsultierte (August 1903) und ich die Pleurahöhle voll von Exsudat fand. In der späteren Krankengeschichte kommt keine Angabe plötzlicher linkseitiger Brustsohmerzen vor; es ist deshalb wahrscheinlich, daß der im August 1907 nachgewiesene Pneumothorex sich nach und nach in dem-

Fig. 1.

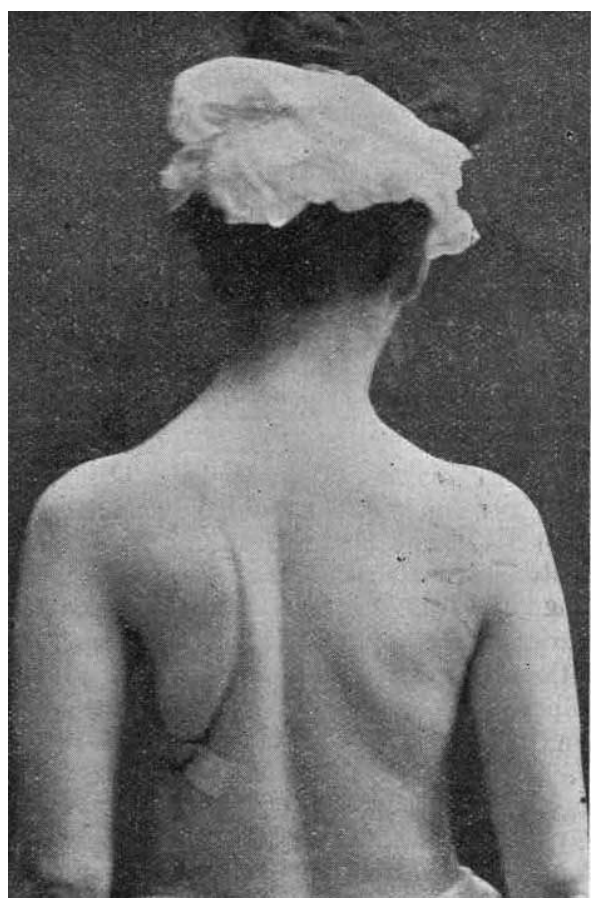

Deutliche linkseitige Skoliose der Dortalregion, schon In der Halsregion beginnend. Die Narbe der Operation den Raud der Scapula entlang. selben Maßstabe gebildet hat, wie das Exsudat der Pleurahöhle resorbiert wurde. Meiner Meinung nach hat sie also vier Jahre lang ihre tägliche Arbeit trotz eines Pneumothorax, der jedenfalls im August 1907 zugleich ein Pyopneumothorax war, besorgt. Am 3. August konnte ich durch eine Probepunktion hinten links Eiter nachweisen.

Dieser Eiter besteht mikroskopisch aus Detritusmasse, in welcher Fettröpfehen und viele große Fettkörnchenzellen, aber keine deutlichen Leukozyten zu sehen sirid. In Deckglaspräparaten können weder Tuberkelbazillen noeh andere Mikroben nachgewiesen werden. Bouillon- und Agar-

kulturen sind nach 48 Stunden immer noch steril. Ein Meerschweinchen wird mit $2 \mathrm{ccm}$ Pus subkutan am Abdomen inokuliert und 11 Wochen später getötet; keine Spur von Tuberkulose.

Durch die gewöhnlichen physikalischen Untersuchungsmethoden war es mir vier Jahre lang unmöglich gewesen, eine richtige Diagnose zu stellen; nur durch die Röntgenstrahlen gelang mir dies zuletzt. Selbst nachdem ich wuBte, daß die Patientin einen Pyopneumothorax haite, konnte ich keine sicheren Zeichen dieses Leidens nachweisen. Nur auf und direkt unterhalb der Clavicula wat der Schall leicht tympanitisch ganz wie bei einer Pleuritis -, sonst nicht, wenn ich auch die Patientin rechte Seiten- oder Bauchlage einmehmen ließ, um dadurch die Stellung der Luft in der Pleurahöhle z⿺ ändern. Wenn ioh sie sohüttelte oder wenn sie schnell und tief Luft holte, konnte ich einige Male unterhalb des Angulus ein fernes, metailisches Glockengeläut hören; ich mußte aber dann mit großer Aufmerksamkeit in längerer Zeit auskultieren. Trotzdem ich den Chef der hiesigen Medizinischen Poliklinik im voraus darauf aufmerksam nachte, daß es sich um etwas Ungewöhnliches handelte, konnte auch er am 5. August 1907 nach der perkutorischen und auskultatorischen Untersuchung derPatientin keine andere Diagnose als ich selbst vor der Röntgenuntersuchung stellen: Tuberkulöse Veränderungen des linken Apex, möglicherweise beginnende Kavernenbildung, im übrigen Zeichen einer alten, geheilten Pleuritis.

Que faire? Die Patientin war trotz ihrer Krankheit bis jetzt arbeitsfähig gewesen. Nichtsdestoweniger schlug ich ihr aus folgenden Gründen eine radikale Operation vor: Selbst wenn das Pleuraexsudat sich durch die Lunge entleert hatte, war dieser Umstand gar kein Beweis dafïr, da $B$ eine davernde Heilung in dieser Weise zu erreichen war; man konnte sich im Gegenteil mit Sicherheit sagen, daß eine Höhle mit so starken Wänden nie ohne Operation heilen und deshalb zu einer fortdauernden Sekretion mit ihren Gefahren (u. a. der Amyloiddegeneration) Veranlassung geben würde. Zweitens mußte man die gesunde rechte Lunge gegen das infizierende Expektorat der linken Lunge und Pleura schützen; durch kein Mittel konnte ich dies besser tun, als indem ich durch eine Operation eine Verödung der Empyemhöhle zu erreichen und dadurch auch die Schrumpfung der Lungenspitze zu erleichtern suchte. Ich machte deshalb am 6. August 1907 die Resektion der zweiten bis elften Rippe.

Chloroformnarkose. Zur Orientierung Resektion von 4-5 cm der neunten Rippe hinten. Es quillt keine Flüssigkeit aus der Pleurahöhle hervor, sondern am Boden derselben sieht man trübe, graue Flüssigkeit, etwa $300 \mathrm{ccm}$, die entleert wird. Die Empyemhöhle erstreckt sich vom Diaphragma bis zu einer Höhe, die ich mit meinem- Finger von der Resektionsöffnung aus nicht erreichen kann. Der Boden der Höhle liegt - in Seitenlage $-7-8 \mathrm{~cm}$ von der Thoraxwand entfernt und wird vom Diaphragma, der stark kollabierten Lunge und dem Pericardium gebildet. Pleura costalis etwas, Pleura pulmonalis enorm verdickt; Tuberkel werden weder jetzt noch später gesehen, ebensowenig eine Perforationsöffnung der Lunge. Weil die Föhle so kolossal war, entschloB ich mich zu der Schedeschen Operation mit dem gewöhnlichen bogenförmigen Schnitt um die Scapula. Subperiostal wurden dann von diesem Schnitte aus alle Rippen mit Ausnahme der eraten und zwölften hinten am Angulus costae, vorne nicht weit vom Rippenknorpel reseziert; das von der zweiten Rippe entfernteStück war jedoch nicht so lang. Die Weichteile des Thorax konnten jetzt ohne wesentliche Spannung mit der Pleura pulmonalis in Berülurung gebraoht werden; teils aus diesem Grunde, hauptsächlich aber weil die Patientin schon ziemlich schwach war, wurden die Weichteile (Pleura costalis, Periost und Muskulatur) nicht entfernt, sondern die Operation sobald wie möghich beendet. Drainage durch den hinteren Sohnitt und durch eine newe Oeffnung dicht am Boden der Pleurahöhle. - Die Operation dauerte mehr als zwei Stunden, Chloroformverbrauch $60 \mathrm{~g}$. Puls nach der Operation 120, weich aber regelmäßig.

In den ersten 24 Stunden war die Kranke sehr unruhig, Puls 110 und 130 , zuweilen sehr klein; während des Verbandwechsels am Morgen nach der Operation kollabierte sie vollständig, erholte sich aber bald wieder. Der weitere Verlauf war ohne Komplikationen, sodaB sie am 1. Oktober, etwa acht Wochen nach der Operation, die Klinik mit einer $4 \times 1,5 \mathrm{~cm}$ großen Fistelöffnung und gutem Allgemeinbefinden zu Fuß verlassen konnte. Einige Wochen später besorgte sie wieder iht Geschäft; nach einem halben Jahr konnte ich keine Rasselgeräusche mehr in der linken Lungenspitze hören, und im Juli 1908, also nach elf Monaten, schloß sich die Fistel vollständig und ist später nie wieder aufgebrochen. Die Patientin ist fortwährend vollständig arbeitsfähig. Sie wurde am 15 . September 1909 in der hiesigen Medizinischen Gesellschaft vorgestellt.

Der Status lautete damals: Blab, das Sprechen ein wenig kupiert. Kein Husten, keine Schmerzen, Appetit gut, fïhlt sich vollständig gesund. Puls 90, $\mathbf{R}, 20$. Die linke Seite der Brust steht wählend der Respiration so gut wie ganz still, die reclyte macht abnorm grole Bewegungen. Die ganze linke Seite ist bedeutend eingesunken, sodaB der linke Arm weiter von der Brustwand entfernt zu sein scheint als der rechte und die linke Schulter. gegend don Findruok macht, daB sie mehr als die rechte prominiert. Die linke Mámma ist bedeutend kleiner als die rechte. Der linke Angulus scapulae tritt stark hervor. Der früheren Fistelöffnung entsprechend ist eine tiefe Finziehung der Weichteile im unteren Teil der Axillarfläche; die Fistel jetzt ganz verschlossen. Von hinten sieht man eine ausgesprochene Skoliose der Dorsal- und der unteren Hals. wirbelsäule, die Konvexität nach links. Brustumfang dicht unter den Armen $67 \mathrm{~cm}$, davon die rechte Seite $38 \mathrm{~cm}$, die linke $29 \mathrm{~cm}$; in Papillarhöhe $67 \mathrm{~cm}$, davon die rechte Seite $38,5 \mathrm{~cm}$, die linke $28,5 \mathrm{~cm}$; unterhalb 
beider Mammae $61 \mathrm{~cm}$, davon die rechte Seite $36 \mathrm{~cm}$, die linke $25 \mathrm{~cm}$. Trotz dieses Mißverhältnisses und trotz der Skoliose ist die Deformation - merkwürdig genug - nicht so in die Augen fallend, daß die Patientin, zum mindesten vollständig bekleidet, in keiner Weise als verunstaltet betrachtet werden kann. - Der linke Arm kann im Schultergelenk beinahe vollständig gehoben werden.

Ueber der ganzen linken Brustseite stark gedämpfter Schall, am stärksten hinten unten. In der Fossa supra- und infraclavicularis samt supraspinata starke Verlängerung der In- und Exspiration; die Respiration sonst beinahe vesikulär und auffallend deutlich bis zur Basis. - Ueber der rechten Lunge hypersonorer Schall, nach unten vorne bis zur achten Rippe, nach links bis zum linken Sternalrand. Auskultatorisch nichts Abnormes. Der Herzsto $\beta$ breit und deutlich in der Axillarfläche, die linke Mamma pulsiert; der Spitzenstoß scheint in dem sechsten oder siebenten Interkostalraum vor dem Rande des Schulterblattes zu liegen. Die Herzdämpfung ist wegen der Dämpfung der linken Lunge nicht sicher festzustellen. Die Herztöne rein.

Bei der Durchleuchtung mit Röntgenstrahlen wird absolut keine respiratorische Bewegung der linken Thoraxhälfte oder der linken Diaphragmahälfte beobachtet, während rechts große Exkursionen gemacht werden. Kein Exsudat in der linken Pleurahöhle; Verdichtungen des Lungengewebes der linken Lungenspitze bis zur dritten Rippe. Ein Röntgenbild zeigt deutlich den kolossalen Defekt der Brustwand nach der Resektion von zehn Rippen; auch daraus kann man schließen, daß die linke Lunge stark geschrumpft und sehr wenig funktionsfähig sein muß. - Urin klar und hell, kein Eiweiß.

SchluB. Dieser Fall scheint mir folgende interessante Punkte zu bieten: Nachdem der erste Schock überstanden ist, geht die Patientin trotz ihrer Pleuritis und ihres Pneumothorax vier Jahre lang ihrer Arbeit nach, ohne einen Tag zu versäumen. Der Pneumothorax mit nachfolgendem Exsudat hat einen - wenn auch vorübergehenden - günstigen Einfluß auf die Spitzenaffektion geübt. Die Diagnose war durch die gewöhnlichen physikalischen Untersuchungsmethoden unmöglich zu stellen, und nur die Durchleuchtung konnte die Gegenwart von Luft und Flïssigkeit in der Pleurahöhle konstatieren. Das Resultat der Operation war ausgezeichnet, wenn auch keine Thorakoplastik, sondern nur eine Resektion der Rippen gemacht wurde; die Fmpyemhöhle obliterierte zuletzt vollständig, und auch die Apexaffektion wurde zur Schrumpfung gebracht. Eine nennenswerte Verunstaltung der Patientin ist durch die Entfernung von zehn Rippen nicht entstanden, was aus den beigefügten Photographien hervorgeht; bei vollständiger Bekleidung sieht man nur eine leichte linkseitige Dorsalskoliose. Auffallend deutlich ist jetzt die Respiration an der linken Hinterfläche zu hören, was als eine Fortleitung von der rechten Seite und kaum als beginnende Funktion der linken Lunge erklärt werden muß; die Durchleuchtung zeigt ja, daß nicht nur die linke Brustwand, sondern auch das Diaphragma der linken Seite während der Respiration ganz still steht. Der Puls ist seit der Operation immer etwa 90, was wohl als ein Ausdruck der wenig günstigen mechanischen Verhältnisse, unter welchen das Herz wegen der veränderten Form des Thorax arbeitet, aufgefaßt werden darf. 